\title{
Accuracy of the non-relativistic approximation to relativistic probability densities for a low-speed weak-gravity system
}

\author{
Shiuan-Ni Liang and Boon Leong Lan ${ }^{\mathrm{a}}$ \\ Electrical and Computer Systems Engineering, School of Engineering, Monash University, 47500 Bandar Sunway, Malaysia
}

Received: 24 November 2014 / Revised: 31 August 2015

Published online: 19 November 2015

(c) The author(s) 2015. This article is published with open access at Springerlink.com

\begin{abstract}
The Newtonian and general-relativistic position and velocity probability densities, which are calculated from the same initial Gaussian ensemble of trajectories using the same system parameters, are compared for a low-speed weak-gravity bouncing ball system. The Newtonian approximation to the general-relativistic probability densities does not always break down rapidly if the trajectories in the ensembles are chaotic — the rapid breakdown occurs only if the initial position and velocity standard deviations are sufficiently small. This result is in contrast to the previously studied single-trajectory case where the Newtonian approximation to a general-relativistic trajectory will always break down rapidly if the two trajectories are chaotic. Similar rapid breakdown of the Newtonian approximation to the generalrelativistic probability densities should also occur for other low-speed weak-gravity chaotic systems since it is due to sensitivity to the small difference between the two dynamical theories at low speed and weak gravity. For the bouncing ball system, the breakdown of the Newtonian approximation is transient because the Newtonian and general-relativistic probability densities eventually converge to invariant densities which are close in agreement.
\end{abstract}

\section{Introduction}

It is conventionally believed [1-4] that, in general, if the speed of a dynamical system is low (i.e., the speed is much less than the speed of light $c$ ) and gravity governs the dynamics but is weak (i.e., the gravitational potential is much less than $c^{2}$ ), the dynamical predictions of general-relativistic mechanics are always well approximated by the Newtonian predictions for the same parameters and initial conditions. However, it was recently [5-7] shown numerically for a bouncing ball system that, although the speed of the ball is low and the gravitational field due to Earth is weak, the Newtonian trajectory will always rapidly disagree completely with the general-relativistic trajectory of the same initial conditions and system parameters if the trajectories are chaotic. The breakdown of agreement is due to sensitivity to the small difference, of order $1 / c^{2}$ (see [6] for details), between the two dynamical theories at low speed and weak gravity, not sensitivity to the initial conditions or system parameters since they are identical in both theories. In contrast, there is no breakdown of agreement between the two predicted trajectories if they are non-chaotic.

The initial conditions of a dynamical system are generally not known precisely in practice and thus a statisticalmechanical approach is necessary to study the dynamics. In this paper, we compare the Newtonian and generalrelativistic position and velocity probability densities, which are calculated from the same initial Gaussian ensemble of trajectories using the same system parameters, for the low-speed weak-gravity bouncing ball system. The aim of this comparison is to determine whether the Newtonian approximation to the general-relativistic probability densities will also always rapidly break down if the trajectories in the ensembles are chaotic, analogous to the chaotic singletrajectory case in [5-7]. Details of our calculations are given next, followed by the presentation and discussion of our results, and finally the conclusion.

\footnotetext{
a e-mail: lan.boon.leong@monash.edu
} 


\section{Methods}

In the bouncing ball system [5-9], a ball repeatedly collides with a vertically oscillating table, which oscillates harmonically with amplitude $A$ and angular frequency $\omega$. The impact between the ball and the table is both instantaneous and inelastic, where the coefficient of restitution $\alpha(0 \leq \alpha<1)$ quantifies the energy loss of the ball at each impact. The table is not affected by the impact because the table's mass is much larger than the ball's mass. In between impacts, the ball moves freely in the gravitational field of the Earth, which is assumed to be a uniform sphere. We will use the ball's velocity $v$ and position $y$ just after each impact to define its trajectory in phase space.

In the Newtonian framework, the dynamics of the bouncing ball is exactly described by the following $2 \mathrm{D}$ map [5-9]:

$$
\begin{aligned}
& A\left[\sin \left(\theta_{k}\right)+1\right]+v_{k}\left[\frac{1}{\omega}\left(\theta_{k+1}-\theta_{k}\right)\right]-\frac{1}{2} g\left[\frac{1}{\omega}\left(\theta_{k+1}-\theta_{k}\right)\right]^{2}-A\left[\sin \left(\theta_{k+1}\right)+1\right]=0, \\
& v_{k+1}=(1+\alpha) \omega A \cos \left(\theta_{k+1}\right)-\alpha\left\{v_{k}-g\left[\frac{1}{\omega}\left(\theta_{k+1}-\theta_{k}\right)\right]\right\}
\end{aligned}
$$

where $v_{k}$ and $\theta_{k}$ are, respectively, the ball's velocity and the table's phase just after the $k$-th impact. The acceleration due to gravity $g=G M / R^{2}$, where $M$ and $R$ are, respectively, the mass and radius of the Earth, and $G$ is the gravitational constant.

In the general-relativistic framework, the dynamics of the bouncing ball is exactly described by the following $2 \mathrm{D}$ $\operatorname{map}[6,7]$ :

$$
\begin{aligned}
& A\left[\sin \left(\theta_{k}\right)+1\right]-A\left[\sin \left(\theta_{k+1}\right)+1\right] \\
& -\frac{c^{2}}{2 g}\left\{1-\frac{2 g\left[R_{\mathrm{TLP}}+A\left[\sin \left(\theta_{k}\right)+1\right]\right]}{c^{2}}\right\}\left\{1-\left\{\frac { 1 } { 2 } \left[\left(1+\beta_{k}\right) e^{-\frac{g}{c}\left(\frac{\theta_{k+1}-\theta_{k}}{\omega}\right)}+\left(1-\beta_{k}\right) e^{\left.\left.\left.\frac{g}{c}\left(\frac{\theta_{k+1}-\theta_{k}}{\omega}\right)\right]\right\}^{-2}\right\}=0}\right.\right.\right. \\
& v_{k+1}=\frac{-c^{2} \alpha\left(\frac{v_{k+1}^{\prime}-u_{k+1}}{c^{2}-v_{k+1}^{\prime} u_{k+1}}\right)+u_{k+1}}{1-\alpha u_{k+1}\left(\frac{v_{k+1}^{\prime}-u_{k+1}}{c^{2}-v_{k+1}^{\prime} u_{k+1}}\right)}
\end{aligned}
$$

where

$$
v_{k+1}^{\prime}=c\left[\frac{\left(1+\beta_{k}\right) e^{-\frac{g}{c}\left(\frac{\theta_{k+1}-\theta_{k}}{\omega}\right)}-\left(1-\beta_{k}\right) e^{\frac{g}{c}\left(\frac{\theta_{k+1}-\theta_{k}}{\omega}\right)}}{\left.\left(1+\beta_{k}\right) e^{-\frac{g}{c}\left(\frac{\theta_{k+1}-\theta_{k}}{\omega}\right)}+\left(1-\beta_{k}\right) e^{\frac{g}{c}\left(\frac{\theta_{k+1}-\theta_{k}}{\omega}\right)}\right]}\right.
$$

is the ball's velocity just before the $(k+1)$-th impact,

$$
u_{k+1}=A \omega \cos \left(\theta_{k+1}\right)
$$

is the table's velocity just after the $(k+1)$-th impact, and $\beta_{k}=v_{k} / c$. The constant $R_{\mathrm{TLP}}$ is the distance between the table's lowest position and the center of the Earth, and $c$ is the speed of light.

The Newtonian map (eqs. (1) and (2)) and the general-relativistic map (eqs. (3) and (4)) each maps the ball's velocity $v$ and the table's phase $\theta$ from just after an impact to just after the next impact. The ball's position $y_{k}$ just after the $k$-th impact is the table's position just after the $k$-th impact, which is easily calculated from the table's phase $\theta_{k}$ just after the $k$-th impact using the equation that describes the vertical sinusoidal oscillation of the table [6,7]:

$$
y_{k}=A\left[\sin \left(\theta_{k}\right)+1\right]
$$

Our derivation of the general-relativistic map follows the same steps as the derivation [8,9] of the Newtonian map, except the free-fall motion of the ball between impacts is treated using general relativity where the gravitational field of the Earth (Earth is assumed to be a uniform sphere) is described by the Schwarzschild metric. We do not assume that the gravitational field is uniform as Lapidus [2] did in his treatment of the general-relativistic free-fall motion. Complete details of our derivation of the general-relativistic map and general-relativistic free-fall motion have been published [6] — see Text S1 and S2, respectively, in the supporting information for the paper (i.e. ref. [6]) - and therefore it is not necessary to present them again here.

In both theories, the ensemble of trajectories is initially Gaussian distributed in position and velocity with means $\left\langle y_{0}\right\rangle$ and $\left\langle v_{0}\right\rangle$, and standard deviations $\sigma_{y_{0}}$ and $\sigma_{v_{0}}$ :

$$
\rho(y, v, t=0)=\frac{1}{2 \pi \sigma_{y_{0}} \sigma_{v_{0}}} \exp \left[-\frac{1}{2}\left[\left(\frac{y-\left\langle y_{0}\right\rangle}{\sigma_{y_{0}}}\right)^{2}+\left(\frac{v-\left\langle v_{0}\right\rangle}{\sigma_{v_{0}}}\right)^{2}\right]\right] .
$$


Each trajectory in the Newtonian (general-relativistic) ensemble is time-evolved in quadruple precision using the Newtonian (general-relativistic) map, where the phase map, eq. (1) (eq. (3)), which is an implicit algebraic equation for $\theta_{k+1}$, must be solved numerically by finding the zero of the function on the left side of the equation, given $\theta_{k}$ and $v_{k}$. We used Brent's method for this purpose [6,7], where the accuracy of the zeroes is specified by an error tolerance. To determine whether a trajectory is chaotic, the largest Lyapunov exponent is calculated numerically using Sprott's version [10] of the standard method - the trajectory is chaotic (i.e., sensitively dependent on initial conditions) if the exponent is positive. The position and velocity probability densities just after each impact are numerically constructed using the trajectories in the ensemble. We first use $10^{6}$ trajectories, calculated with $10^{-30}$ error tolerance for the ball's position, to construct each probability density, and check its accuracy by comparing it with the probability density constructed using the same number of trajectories calculated with a smaller error tolerance of $10^{-32}$. The probability density is then re-calculated using $10^{7}$ trajectories with $10^{-30}$ error tolerance and its accuracy is checked in the same way. Finally, the accuracy of the $10^{6}$-trajectory probability density is checked by comparing it with the $10^{7}$-trajectory probability density.

\section{Results}

We will now present two representative examples to illustrate our finding. In both examples, the coefficient of restitution $\alpha$ is 0.5 , and we used $g=981 \mathrm{~cm} / \mathrm{s}^{2}, c=3 \times 10^{10} \mathrm{~cm} / \mathrm{s}$, and $R_{\mathrm{TLP}}=6.4 \times 10^{8} \mathrm{~cm}$ (mean radius of the Earth) for the constants. The mean velocity of the trajectories in each ensemble remained low, about $10^{-10} c\left(i . e ., 10^{-8}\right.$ percent of the speed of light $c$ ), and the gravitational field is weak, about $10^{-9} c^{2}$.

In the first example, the amplitude $A$ and frequency $\omega / 2 \pi$ of the table's oscillation are, respectively, $0.012 \mathrm{~cm}$ and $60 \mathrm{~Hz}$. The ensemble of trajectories in both theories is initially Gaussian distributed in position and velocity with means $\left\langle y_{0}\right\rangle=0.02022 \mathrm{~cm}$ and $\left\langle v_{0}\right\rangle=8.17001 \mathrm{~cm} / \mathrm{s}$, and standard deviations $\sigma_{y_{0}}=10^{-10} \mathrm{~cm}$ and $\sigma_{v_{0}}=10^{-10} \mathrm{~cm} / \mathrm{s}$. In this case, although the trajectories in both ensembles are chaotic, there is no breakdown of agreement between the Newtonian and general-relativistic position and velocity probability densities — fig. 1 illustrates this for impacts 25 and 48.

In the second example, the two parameters for the table's oscillation are the same as the first example. For the initial Gaussian ensemble, the position and velocity means are also the same as the first example, but the standard deviations are smaller, i.e., $10^{-13} \mathrm{~cm}$ and $10^{-13} \mathrm{~cm} / \mathrm{s}$, respectively. The trajectories in both ensembles are also chaotic, with positive Lyapunov exponents. Furthermore, for each theory, the close similarity between the phase-space plots (see figs. 2 and 3, respectively, for the general-relativistic and Newtonian case) for the ensemble (from impacts 0 to 71) and a single chaotic trajectory with initial conditions equal to the initial means of the ensemble (from impacts 0 to 2000) show that the ensemble is evolving on a chaotic attractor. The ensemble, which is initially seen as a dot at $(0.02022,8.17001)$ in the phase-space plot, converges (because of dissipation due to inelastic impacts) very rapidly - after two impacts - to the chaotic attractor as it spreads out. The Newtonian and general-relativistic attractors plotted in phase space are not exactly the same but close because of the small difference between the two dynamical theories at such low speed and weak gravity.

In the second example, in contrast to the first example, the general-relativistic position and velocity probability densities are not always well approximated by the corresponding Newtonian probability densities, the breakdown of agreement occurs rapidly, at impact 55. At this impact, the Newtonian and general-relativistic probability densities, for both position and velocity, are approximately still Gaussians but with larger widths (see fig. 4). However, the center and width of the Newtonian and general-relativistic probability densities are different. After this impact, the probability densities continue to spread - fig. 4 shows the different Newtonian and general-relativistic position and velocity probability densities at impact 71 when the position densities have spread out maximally and the densities are no longer Gaussians. In contrast, in the first example, fig. 1 shows there is no breakdown of agreement between the Newtonian and general-relativistic position and velocity probability densities even when the position densities have spread out maximally and the densities are no longer Gaussians at impact 48.

In figs. 1 and 4 , the four calculations $\left(10^{6}\right.$-trajectory $10^{-30}$-error tolerance, $10^{6}$-trajectory $10^{-32}$-error tolerance, $10^{7}$-trajectory $10^{-30}$-error tolerance, and $10^{7}$-trajectory $10^{-32}$-error tolerance) of each probability density predicted by a theory are indistinguishable in the plot. Thus the breakdown of agreement between the Newtonian and generalrelativistic probability densities in the second example, shown in fig. 4, is not a numerical artifact.

The breakdown of agreement in the second example can be understood as follows. Firstly, in each theory, before the position probability density is spread out maximally (at impact 71 and 68, respectively, in the Newtonian and general-relativistic case), the centers (measured by the means) of the position and velocity probability densities, which are approximately Gaussians, are well approximated by the chaotic trajectory generated by the 2D map using the same initial conditions as the centers - see figs. 5 and 6 . These two chaotic trajectories, Newtonian and generalrelativistic, which coincide initially since the centers of the Newtonian and general-relativistic probability densities coincide initially, have positive Lyapunov exponents that are close, about 0.34 [6]. Moreover, they diverge exponentially, on average, from each other (the exponential growth constant of the distance between the two trajectories is close 

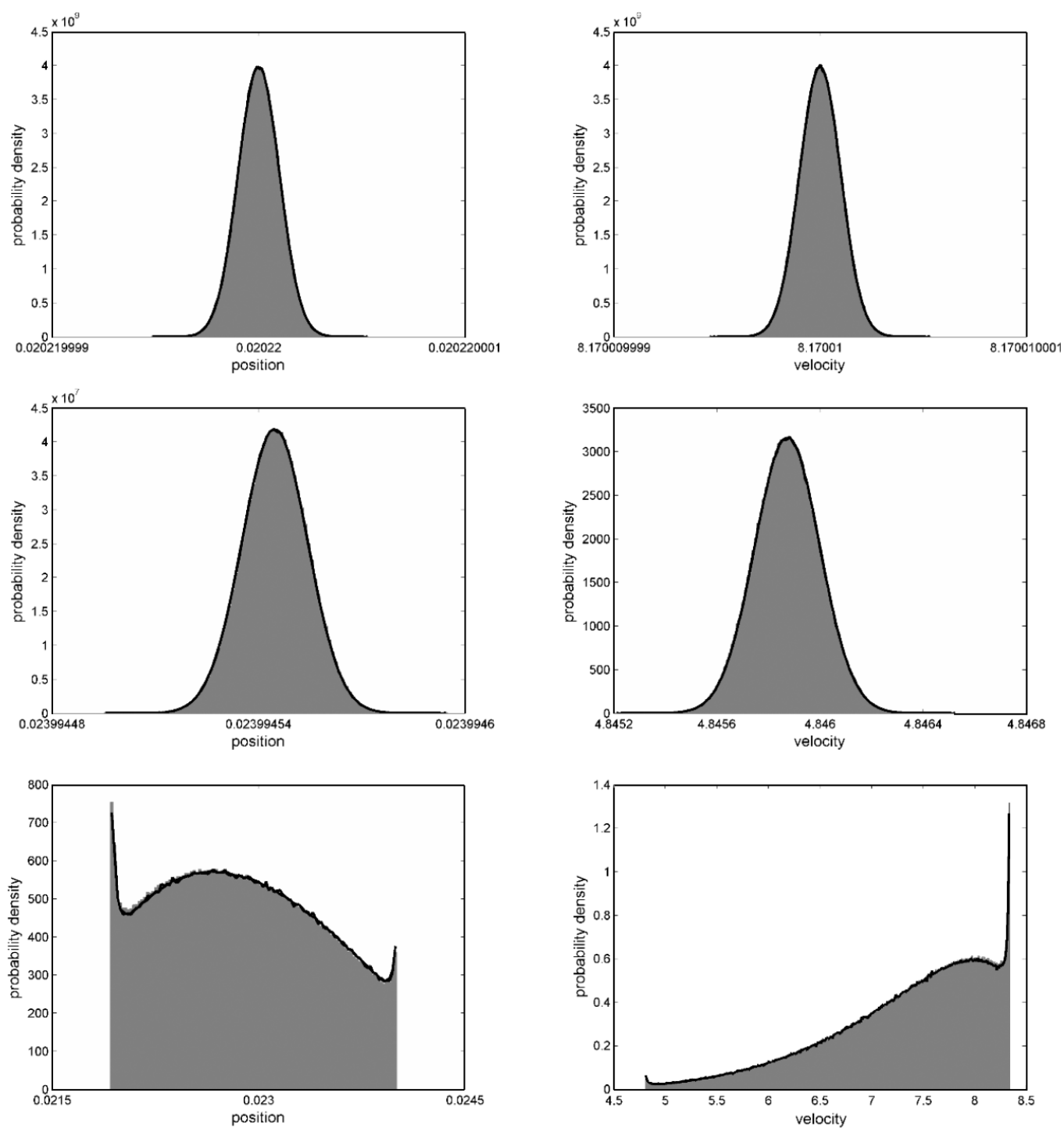

Fig. 1. Newtonian (grey) and general-relativistic (white) position (left column) and velocity (right column) probability densities for the first example at impacts 0 (first row), 25 (middle row) and 48 (last row).

to the Lyapunov exponents of the trajectories) because of the small difference between the Newtonian and generalrelativistic maps at low speed and weak gravity, and disagree completely at impact 55 (see [6]). Therefore the centers of the Newtonian and general-relativistic probability densities also diverge exponentially, on average, from each other and disagree completely when the two Newtonian and general-relativistic chaotic trajectories disagree completely at impact 55 .

Secondly, in each theory, before the position probability density is spread out maximally, the widths (measured by the variances) of the position and velocity probability densities grow, on average, exponentially (see fig. 7), where the exponential growth constants are close to two times the Lyapunov exponent of the chaotic trajectory with the same initial conditions as the centers. However, the exponential growth constants of the Newtonian and general-relativistic widths (0.6865 and 0.6982 , respectively, for position variance, 0.7034 and 0.7235 , respectively, for velocity variance) are close, with a small difference $\delta$, but not identical because of the small difference between the Newtonian and general-relativistic maps, which are used to time-evolve the corresponding ensembles, at low speed and weak gravity. Consequently, the Newtonian and general-relativistic widths (for position or velocity), which are the same initially, increasingly diverge with impact $n$

$$
\operatorname{var}_{G R}-\operatorname{var}_{N} \approx \operatorname{var}_{0}\left[e^{(a+\delta) n}-e^{a n}\right] \approx \operatorname{var}_{0} \delta n e^{a n}
$$



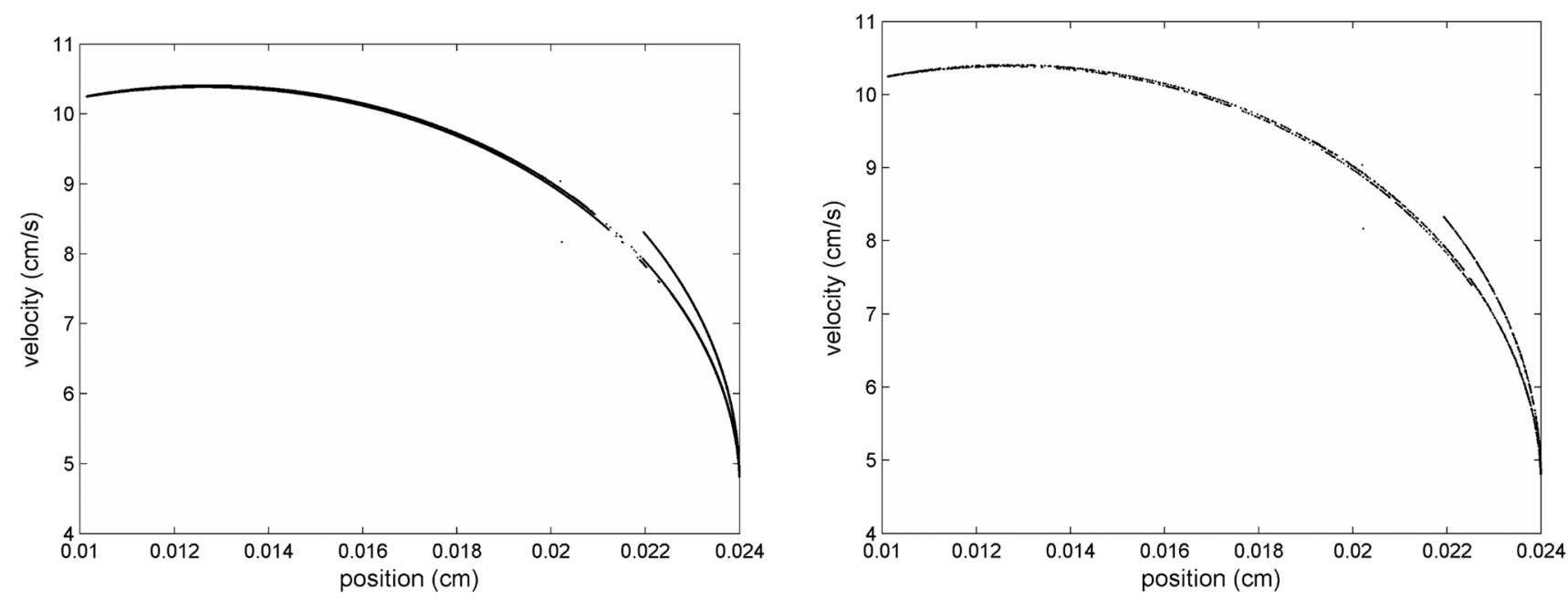

Fig. 2. Phase-space plot for the general-relativistic ensemble $\left(10^{6}\right.$ trajectories $)$ from impacts 0 to 71 (left) and a single chaotic trajectory (with initial conditions equal to the initial means of the ensemble) from impacts 0 to 2000 (right).
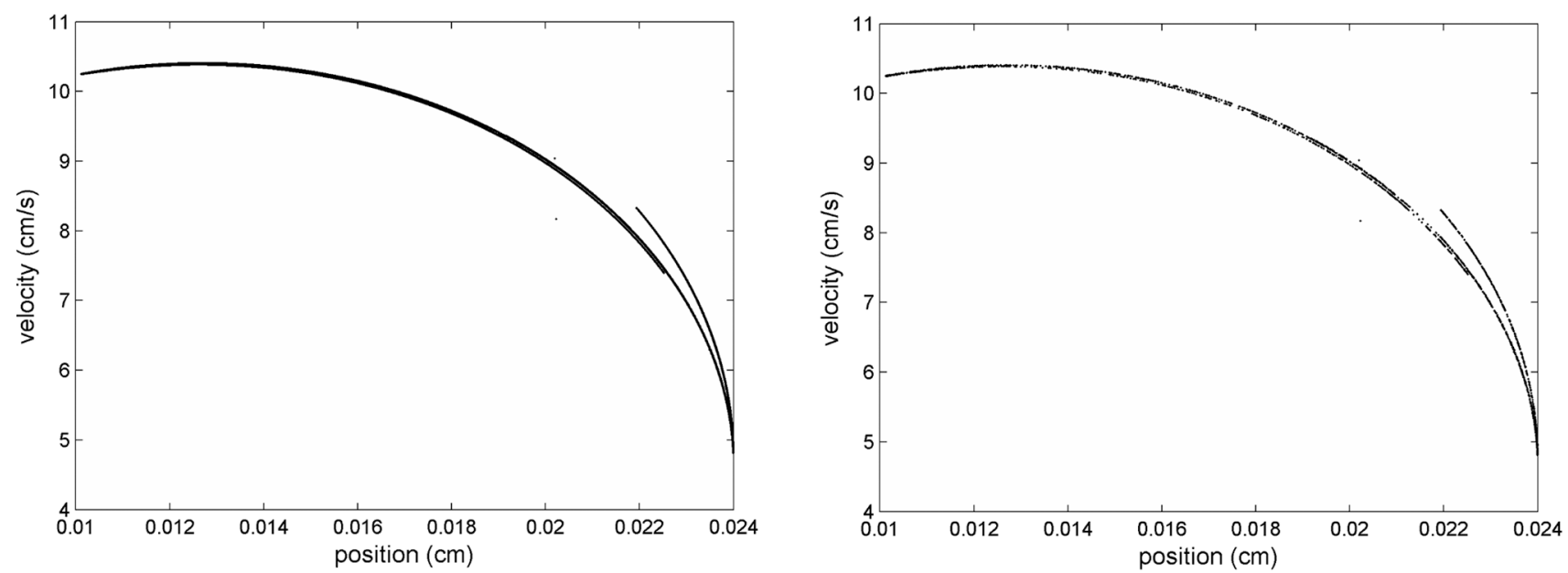

Fig. 3. Phase-space plot for the Newtonian ensemble ( $10^{6}$ trajectories) from impacts 0 to 71 (left) and a single chaotic trajectory (with initial conditions equal to the initial means of the ensemble) from impacts 0 to 2000 (right).

and disagree completely, as we have found, at impact 52. In the expression above for the difference between the generalrelativistic and Newtonian variances as a function of impact $n, \operatorname{var}_{0}$ is the initial variance and $a$ is the exponential growth constant of the Newtonian variance.

In the first example, the Newtonian and general-relativistic centers and widths also diverge in similar manners but, in contrast to the second example, the position probability densities are spread out maximally earlier, at impact 48, before the breakdown of agreements could occur.

For both theory in the second example, after the position density has spread out maximally, the position and velocity probability densities eventually converge to stationary or invariant densities because of dissipation. Figure 8 shows that the Newtonian and general-relativistic invariant densities are close for both position and velocity.

\section{Conclusion}

The two examples for the bouncing ball system illustrate that the agreement between the Newtonian and generalrelativistic initially Gaussian position and velocity probability densities, where the densities are constructed from ensembles of chaotic trajectories, breaks down rapidly only if the initial position and velocity standard deviations are sufficiently small. Like the chaotic single-trajectory case [6], this rapid breakdown of the Newtonian approximation is also due to sensitivity to the small difference between the two dynamical theories at low speed and weak gravity since the initial ensemble of trajectories and system parameters are identical in both theories. Because the small 

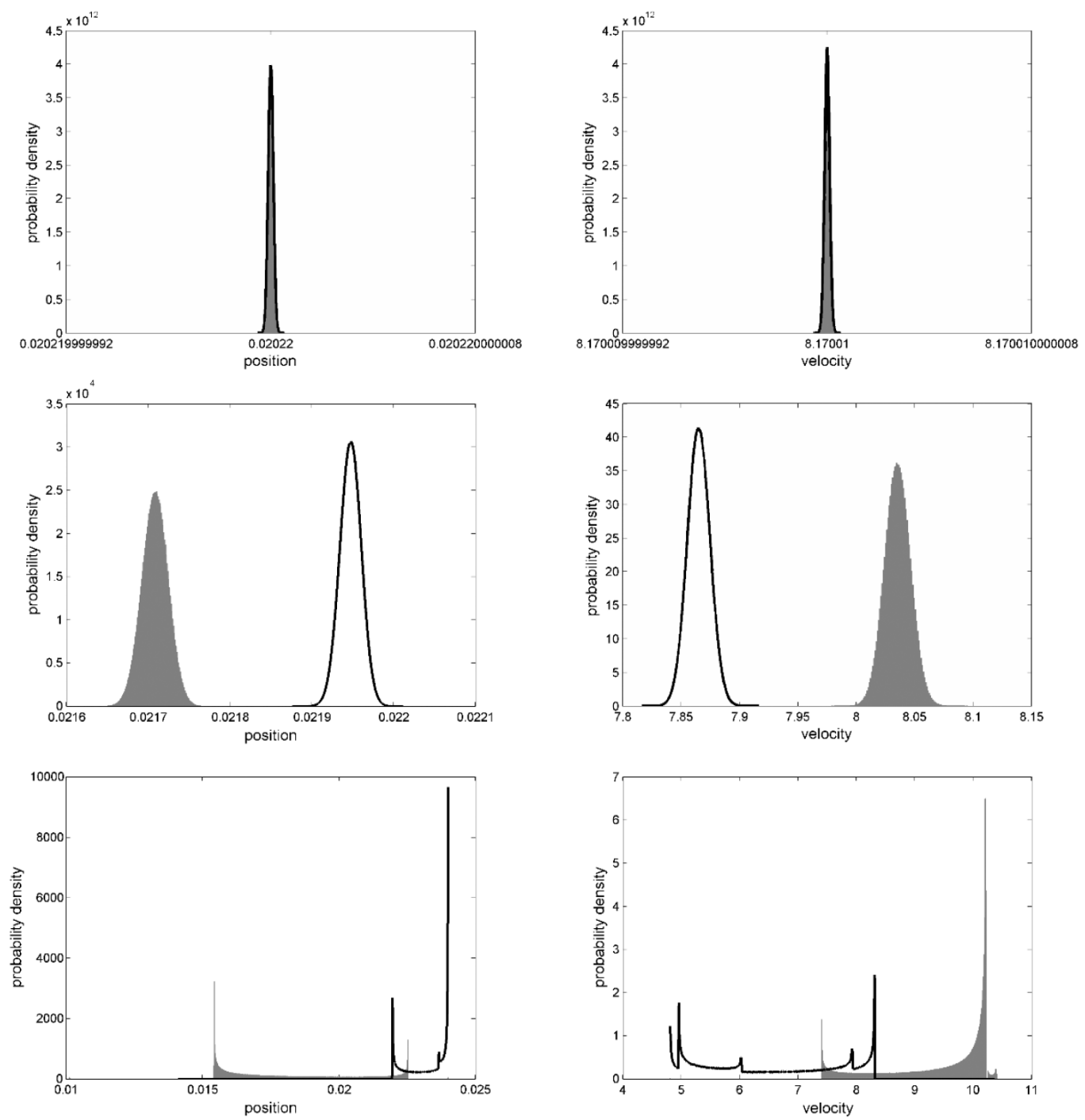

Fig. 4. Newtonian (grey) and general-relativistic (white) position (left column) and velocity (right column) probability densities for the second example at impacts 0 (first row), 55 (middle row) and 71 (last row).

difference between the two dynamical theories at low speed and weak gravity is generic, similar breakdown of the Newtonian approximation to the general-relativistic probability densities should also occur for other low-speed weakgravity chaotic systems, for example, a gravitational three-body system. However, the breakdown of the Newtonian approximation is only transient in the dissipative bouncing ball system - eventually the Newtonian and generalrelativistic probability densities converge to invariant densities that are close in agreement. In other words, although the Newtonian approximation to the general-relativistic probability densities can be unreliable in the short term, it is a good approximation in the long term.

This work was funded by a Fundamental Research Grant FRGS/1/2013/ST02/MUSM/02/1. Part of the work was done by BLL at the Institute of Physics, Faculty of Science, University of the Republic, Uruguay, as a TWAS-Unesco Associate.

Open Access This is an open access article distributed under the terms of the Creative Commons Attribution License (http://creativecommons.org/licenses/by/4.0), which permits unrestricted use, distribution, and reproduction in any medium, provided the original work is properly cited. 

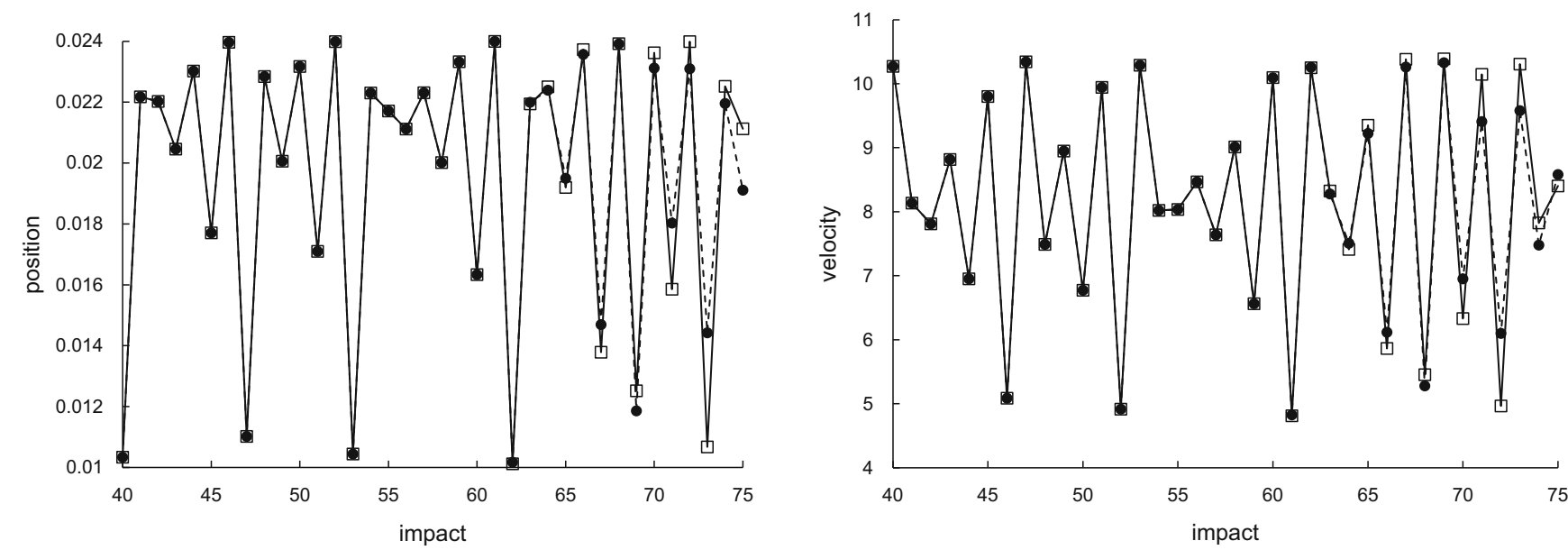

Fig. 5. Newtonian single trajectory (squares) and means of the Newtonian probability densities (circles) for the second example. The data before impact 40 is not plotted in each graph because the two quantities are still close to each other.
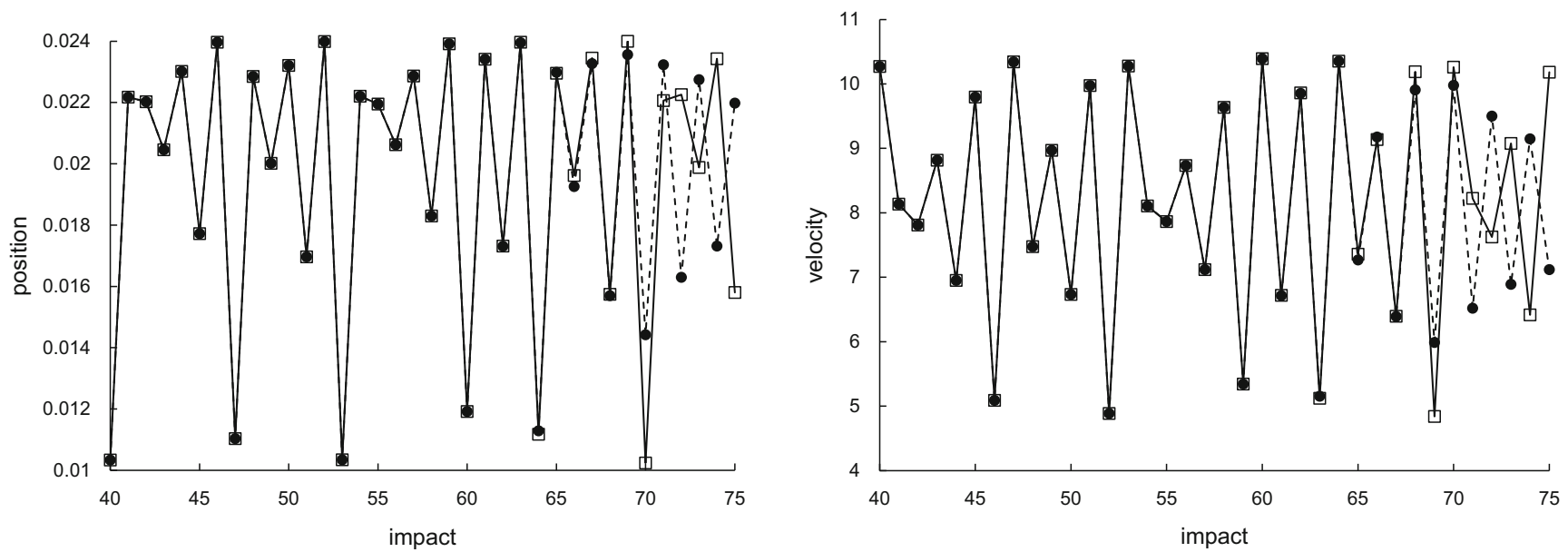

Fig. 6. General-relativistic single trajectory (squares) and means of the general-relativistic probability densities (circles) for the second example. The data before impact 40 is not plotted in each graph because the two quantities are still close to each other.
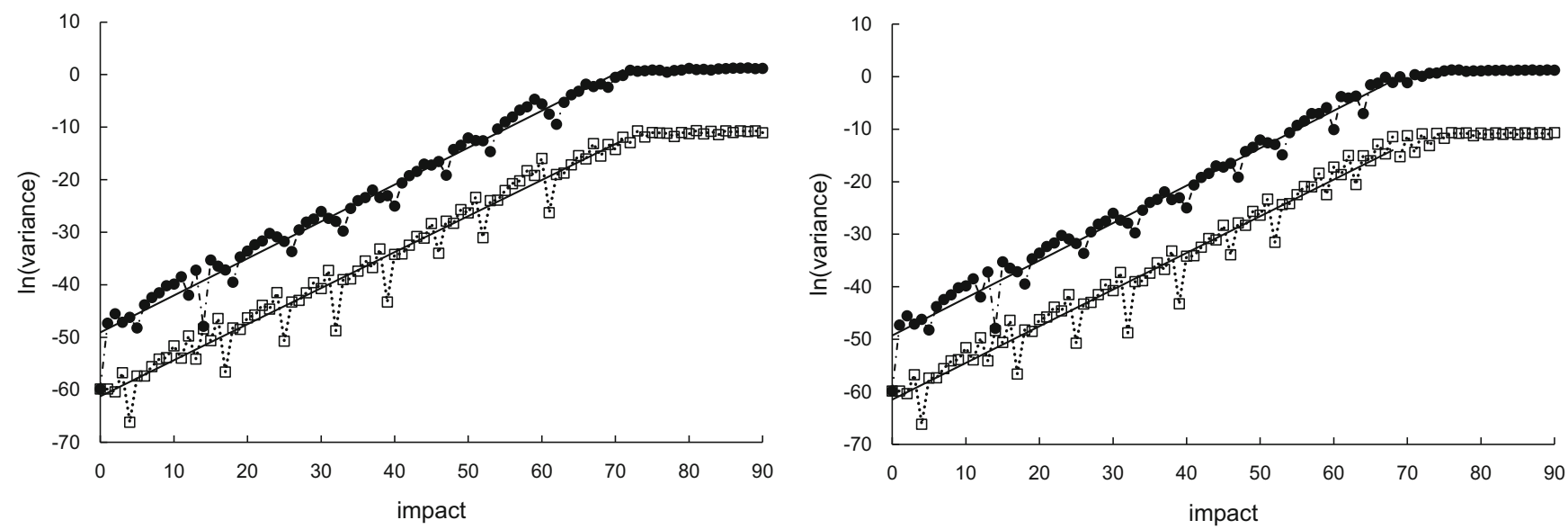

Fig. 7. Natural-log of the Newtonian (left) and general-relativistic (right) position (squares) and velocity (circles) variances for the second example. Straight-line fits up to impact 71 and 68 are also plotted, respectively, in the left and right panels. The Newtonian and general-relativistic position densities are spread out maximally at these impacts, respectively. 

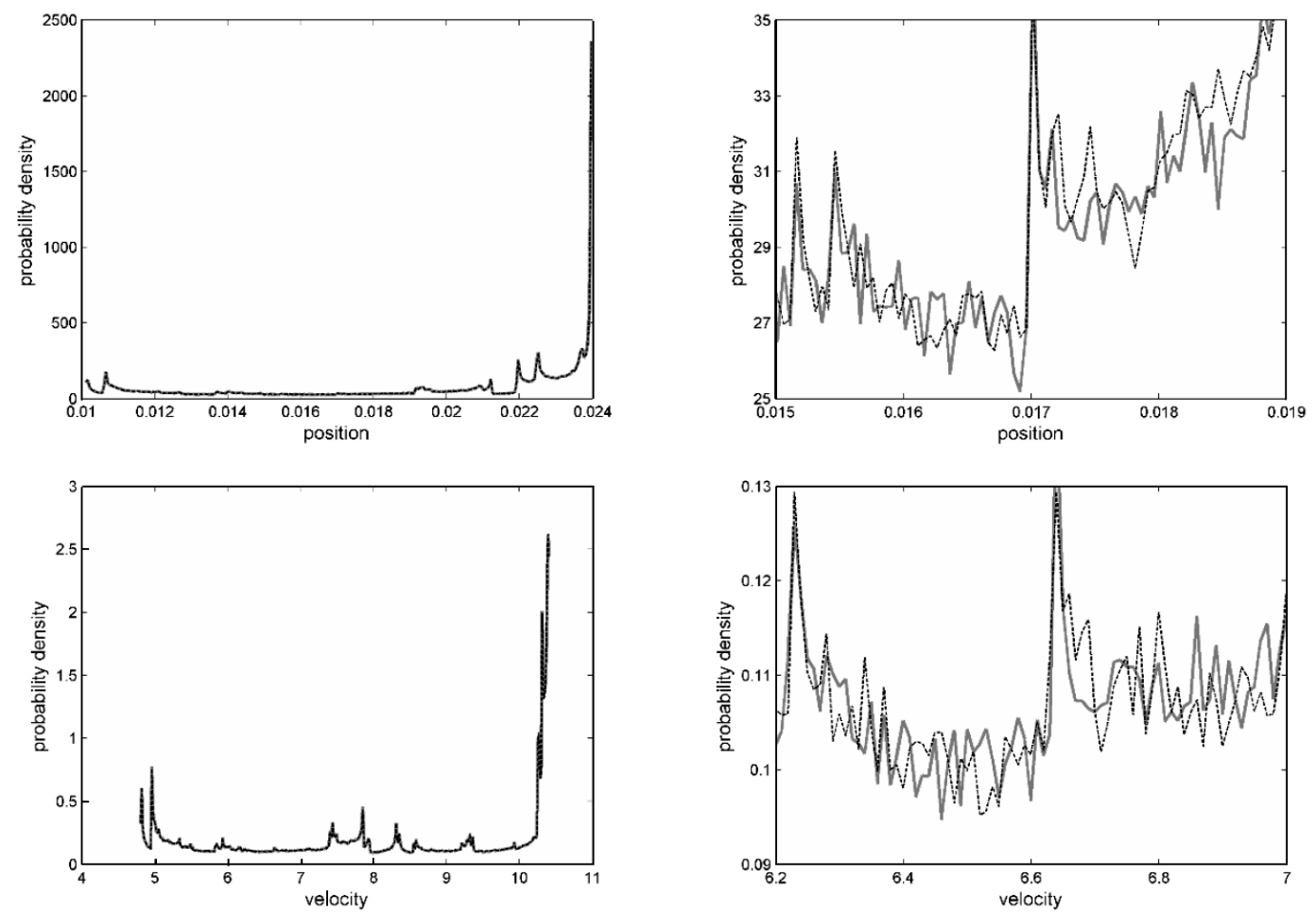

Fig. 8. Newtonian (grey solid line) and general-relativistic (black dashed line) probability densities for position (first row) and velocity (second row) at impact 200: full view (left column) and enlarged view of some parts (right column).

\section{References}

1. A. Einstein, Relativity: the Special and the General Theory (Random House, New York, 1961).

2. I.R. Lapidus, Am. J. Phys. 40, 1509 (1972).

3. R.M. Wald, General Relativity (University of Chicago Press, Chicago, 1984).

4. Y. Choquet-Bruhat, General Relativity and the Einstein Equations (Oxford University Press, New York, 2009).

5. S.N. Liang, B.L. Lan, Results Phys. 1, 36 (2011).

6. S.N. Liang, B.L. Lan, PLOS One 7, e34720 (2012).

7. S.N. Liang, B.L. Lan, Results Phys. 4, 187 (2014).

8. N.B. Tufillaro, T.M. Mello, Y.M. Choi, A.M. Albano, J. Phys. (Paris) 47, 1477 (1986).

9. N.B. Tufillaro, T. Abbott, J. Reilly, An Experimental Approach to Nonlinear Dynamics and Chaos (Addison-Wesley, California, 1992).

10. J.C. Sprott, Chaos and Time-series Analysis (Oxford University Press, Oxford, 2003). 